\title{
Genetic overgrowth syndrome: A single center's experience
}

\author{
Chong Kun Cheon", ${ }^{1}$, Yoo-Mi Kim², Ju Young Yoon ${ }^{1}$, and Young A Kim ${ }^{1}$ \\ ${ }^{1}$ Department of Pediatrics, Pusan National University Children's Hospital, Pusan National University School of Medicine, Yangsan, Korea \\ ${ }^{2}$ Department of Pediatrics, Chungnam National University Hospital, Chungnam National University College of Medicine, Daejeon, Korea
}

Purpose: Overgrowth syndromes are conditions that involve generalized or localized areas of excess growth. In this study, the clinical, molecular, and genetic characteristics of Korean patients with overgrowth syndrome were analyzed.

Materials and Methods: We recruited 13 patients who presented with overgrowth syndrome. All patients fulfilled inclusion criteria of overgrowth syndrome. Analysis of the clinical and molecular investigations of patients with overgrowth syndrome was performed retrospectively.

Results: Among the 13 patients with overgrowth syndrome, 9 patients (69.2\%) were found to have molecular and genetic causes. Among the seven patients with Sotos syndrome (SS), two had a $5 q 35$ microdeletion that was confirmed by fluorescent in situ hybridization. In two patients with SS, intragenic mutations including a novel mutation, c.5993T>A (p.M1998L), were found by Sanger sequencing. One patient had one copy deletion of NDS1 gene which was confirmed by multiplex ligationdependent probe amplification. Among five patients with Beckwith-Wiedemann syndrome, three had aberrant imprinting control regions; 2 hypermethylation of the differentially methylated region of $\mathrm{H} 19,1$ hypomethylation of the differentially methylated region of $\mathrm{Kv}$. In one patient displaying overlapping clinical features of SS, a de novo heterozygous deletion in the chromosomal region 7q22.1-22.3 was found by single nucleotide polymorphism-based microarray.

Conclusion: Considering high detection rate of molecular and genetic abnormalities in this study, rigorous investigations of overgrowth syndrome may be an important tool for the early diagnosis and genetic counseling. A detailed molecular analysis of the rearranged regions may supply the clues for the identification of genes involved in growth regulation.

Key words: Growth, Sotos syndrome, Beckwith-Wiedemann syndrome, Early diagnosis.

\section{Introduction}

Overgrowth syndromes are a heterogeneous group of conditions characterized by either localized or generalized excessive growth for age and sex. Most overgrowth syndromes comprise a group of disorders associated with excessive growth and other features, such as facial dysmorphism, congenital anomalies, neurological problems and an increased risk of neoplasia [1]. Therefore, it is important to diagnose the overgrowth syndromes for accurate genetic counseling and to inform cancer surveillance and prognosis prediction. However, diagnostic categorization of these conditions is difficult because many have overlapping clinical features. Recent advances in understanding the genetic basis of overgrowth syndromes have resulted in a move

Received: 25 May 2018, Revised: 1 August 2018, Accepted: 3 August 2018, Published: 31 December 2018

${ }^{*}$ Corresponding author: Chong Kun Cheon, M.D., Ph.D. (iD http://orcid.org/0000-0002-8609-5826

Department of Pediatrics, Pusan National University Children's Hospital, Pusan National University School of Medicine, 20 Geumo-ro, Mulgeum-eup, Yangsan 50612, Korea.

Tel: +82-55-360-3158, Fax: +82-55-360-2181, E-mail: chongkun@pusan.ac.kr

Conflict of interest: The authors declare that they do not have any conflicts of interest.

(C) This is an open-access article distributed under the terms of the Creative Commons Attribution Non-Commercial License (http://creativecommons.org/licenses/by-nc/4.0/) which permits unrestricted non-commercial use, distribution, and reproduction in any medium, provided the original work is properly cited.

(c) Copyright 2018 by the Korean Society of Medical Genetics and Genomics 
away from clinical classification to molecular classification of overgrowth syndromes [2]. Despite these recent advances, the disease causing mechanism remains unknown in $20-40 \%$ of patients, underlining the genetic heterogeneity of these disorders [1]. Moreover, many patients with overgrowth syndromes do not belong to any recognizable condition and remain unexplained. In this study, we report 13 patients with overgrowth syndromes including recognizable overgrowth syndrome and unclassified overgrowth syndrome. In addition to their clinical relevance, our results emphasize the importance of a chromosomal imbalance in the etiology of unclassified overgrowth syndrome and may help to identify novel genes involved in growth anomalies.

\section{Materials and Methods}

\section{Ethics statement}

This study was performed in accordance with the Declaration of Helsinki. Appropriate institutional review board approval was obtained for this study from the Institutional Review Board at Pusan National University Yangsan Hospital (IRB no. 05-2018069). Written informed consent was obtained from each participant before the collection of blood samples and subsequent analysis.

\section{Study patients and molecular analysis}

A total of 20 patients suspected of overgrowth syndrome visited to Pusan National University Children's Hospital from March 2009 to March 2016. Among them, 13 patients (7 females and
6 males) were evaluated to identify the underlying causes of overgrowth syndrome and were enrolled in this study. Flow sheet showing a diagnostic approach to generalized overgrowth is depicted in Fig. 1. A loose criteria of overgrowth syndrome was used in this study because there might be considerable phenotypic and etiologic heterogeneity in this domain. For example, approximately $10 \%$ of the children with Sotos syndrome (SS) have a height $<97$ th percentile [3]. A loose criteria includes height $>95$ th percentile and/or head circumference $>97$ th percentile, dysmorphic craniofacial features, and/or congenital anomalies. All patients fulfilled inclusion criteria of overgrowth syndrome. Clinical criteria of SS included a distinctive facial appearance (broad and prominent forehead, sparse frontotemporal hair, downslanting palpebral fissures, long and narrow face, long chin), developmental delay (DD), mild to severe intellectual disability (ID), and overgrowth (head circumference $\geq 2$ standard deviation [SD] above the mean). For the diagnostic approach to SS, fluorescent in situ hybridization (FISH) study was initially performed on metaphase spreads of cultured peripheral leukocytes stimulated with phytohemagglutinin using an NSD 1 probe (Vysis LSI NSD 1; Abbott Laboratories, Chicago, IL, USA) on 5q35. If the FISH analysis revealed no deletion, NSD1 sequencing analysis was performed to identify intragenic mutations successively followed by multiplex ligation-dependent probe amplification (MLPA). All coding exons and exon-intron boundaries of the NSD1 gene were amplified by polymerase chain reaction (PCR) and a thermal cycler (Applied Biosystems, Foster City, CA, USA). Direct sequencing was done using a BigDye Terminator

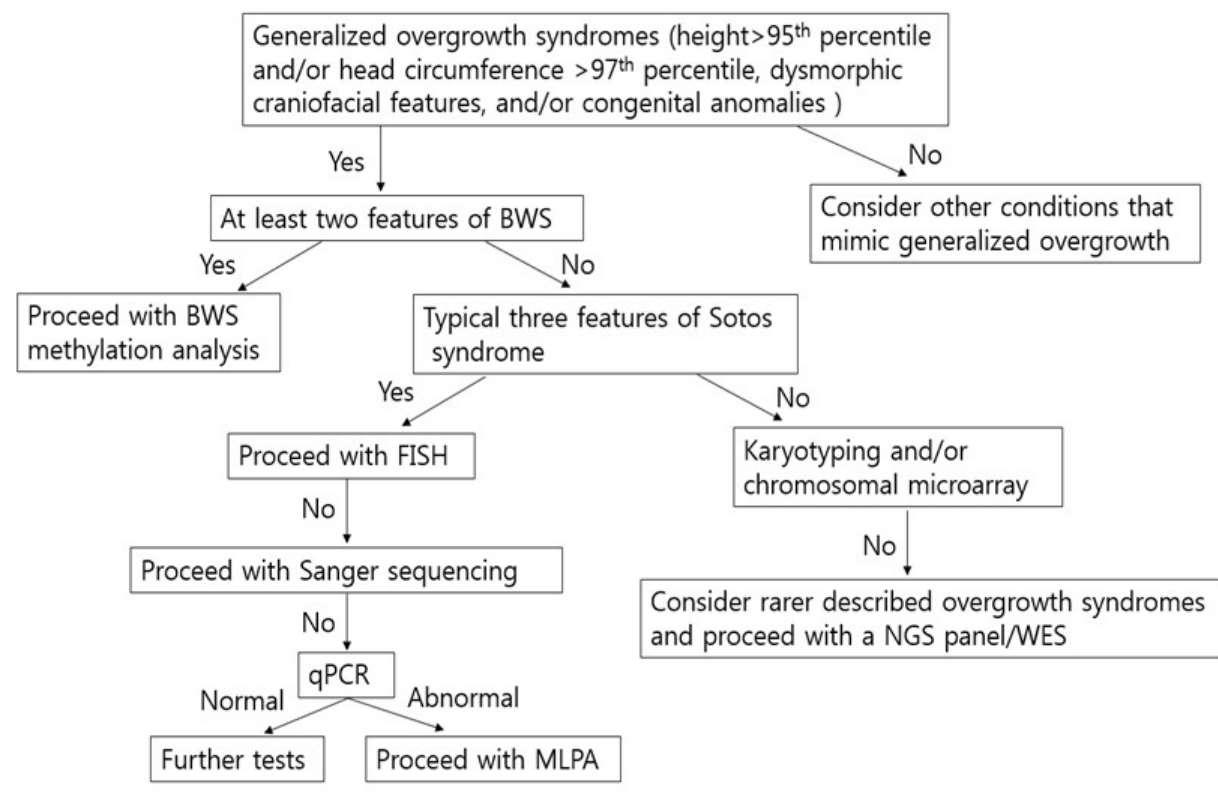

Fig. 1. Flow chart showing a diagnostic approach to generalized overgrowth. BWS, Beckwith-Wiedemann syndrome; FISH, fluorescent in situ hybridization; NGS, next generation sequencing; WES, whole exome sequencing; PCR, polymerase chain reaction; MLPA, multiplex ligation-dependent probe amplification. 
Cycle Sequencing Ready Reaction kit (Applied Biosystems), and the analysis was performed using an ABI3130xI Genetic Analyzer (Applied Biosystems).

Cardinal features of Beckwith-Wiedemann syndrome (BWS) include macrosomia, macroglossia, hemihyperplasia, and ear creases/pits. Methylation analysis of the 2 imprinted domains at 11 p15.5 (H19DMR and differentially methylated region of Kv [KvDMR]) was performed by methylation-specific PCR in the patients presenting with at least two features of BWS. When recognizable overgrowth syndrome such as SS and BWS were ruled out based on clinical assessment, we performed karyotyping/single nucleotide polymorphism (SNP)-based microarray (Affymetrix Cytoscan 750K array [Affymetrix, Santa Clara, CA, USA]) analysis as first step for unclassified overgrowth syndrome. Clinical manifestations, clinical courses, and results of molecular and genetic study were retrospectively reviewed.

\section{Results}

\section{Auxological data and molecular analysis for patients with overgrowth syndrome}

Thirteen patients were diagnosed with overgrowth syndrome during a period of seven years. Detailed demographics of the enrolled patients are presented in Table 1. Seven (53.8\%) were female and six (46.2\%) were male. The patients had mean \pm SD gestational age of $37.7 \pm 3.3$ weeks (range, 30.6 to 43.0 weeks) and mean \pm SD birth weight standard deviation score of $0.002 \pm 0.001$. Age at the time of overgrowth syndrome diagnosis was $30.2 \pm 24.0$ months (range, 0.3 to 84.0 months). Among 13 patients, seven (53.8\%) were diagnosed with SS followed by five (38.5\%) patients with BWS, and one (7.7\%) with 7q22.1$7 q 22.3$ microdeletion. All cases were sporadic. Of these 13 patients, seven (53.8\%) had more than 97th percentile height, six (46.1\%) had more than 97th percentile body weight, and eight (61.5\%) had more than 97th percentile head circumference at diagnosis. Among the seven patients with SS, two had a $5 q 35$ microdeletion that was confirmed by FISH. A novel missense mutation, c.5993T>A (p.M1998L), and a nonsense mutation, c. $5581 \mathrm{C}>\mathrm{T}\left(\mathrm{p} . \mathrm{R} 1861^{*}\right)$, were found in two patients with SS by Sanger sequencing. One patient had one copy deletion of NDS1 gene including a small part of FGFR 4 which was confirmed by MLPA. Two patients were diagnosed with probable SS without any mutation or deletion. Among five patients with BWS, three had aberrant imprinting control regions; 2 hypermethylation of the differentially methylated region of H19 (H19DMR), 1 hypomethylation of the KvDMR. Two patients were diagnosed with probable BWS without any abnormal methylation. In one patient showing normal karyotype of peripheral leukocytes, a de novo heterozygous $2.2 \mathrm{Mb}$ deletion in the chromosomal region 7q22.1-22.3 was found by SNP-based microarray. The deletion started from 102,877,293 bp extending to 105,121,326 bp [Genomic build: $\mathrm{Hg} 19]$. The deleted region contains nine genes: PMPCB, DNAJC2, PSMC2, SLC26A5, RELN, ORC5L, LHFPL3, $K M T 2 E$, and $S P R K 2$ (Fig. 2).

\section{Clinical manifestations of patients with overgrowth syndrome}

All of the patients suspected as overgrowth syndrome had excessive growth, facial dysmorphism, and congenital anomalies at the time of their clinic visit. Among the seven SS patients, three displayed scoliosis and had epilepsy that required antiepileptic drug treatment (Fig. 3). Three patients had abnormal brain regions that included periventricular leukomalacia (PVL), microadenoma at the left lobe of the pituitary gland, cavum septum pellucidum, and ventriculomegaly. Two patients with SS had attention deficit hyperactivity disorder and renal anomaly including left renal pelviectasis and multicystic dysplastic kidney, respectively. One patient each had cardiac anomaly such as atrial septal defect (ASD), hyperinsulinemic hypoglycemia, and umbilical hernia. All patients with BWS had macroglossia. Among the five patients with BWS, three had hemihypertrophy which was left-side dominant, two patients had an umbilical hernia and hypoglycemia during infancy, respectively (Fig. 4). One patient with hypomethylation of KvDMR was diagnosed with central precocious puberty requiring gonadotropin releasing hormone agonist, complex partial seizure, PVL, and focal stenosis of the aqueduct. One patient with hypermethylation of H19DMR had left hepatoblastoma and renal anomaly that included renal pelviectasis. Finally, a 3-year-old-boy had 7q22.1$7 q 22$ microdeletion. He was the second child of unrelated healthy parents of normal stature. Dysmorphic features included high and prominent forehead, hypertelorism, and small mouth. Other features noted included cryptorchidism, retractile testis, recurrent hypoglycemia during infancy, and DD. The patient displayed a vigorous appetite at one-year-of-age. Later, he developed severe obesity (body mass index $>99$ th percentile). He began to walk unaided at 23-months-of-age and could only speak one word at 3-years-of-age. To date, at 3 years, his height, weight and head circumference are over 97 th percentile. Bone age is advanced over chronological age by 6 months. Brain magnetic resonance imaging (MRI) revealed hypoplasia of the corpus callosum and cortical dysplasia. 


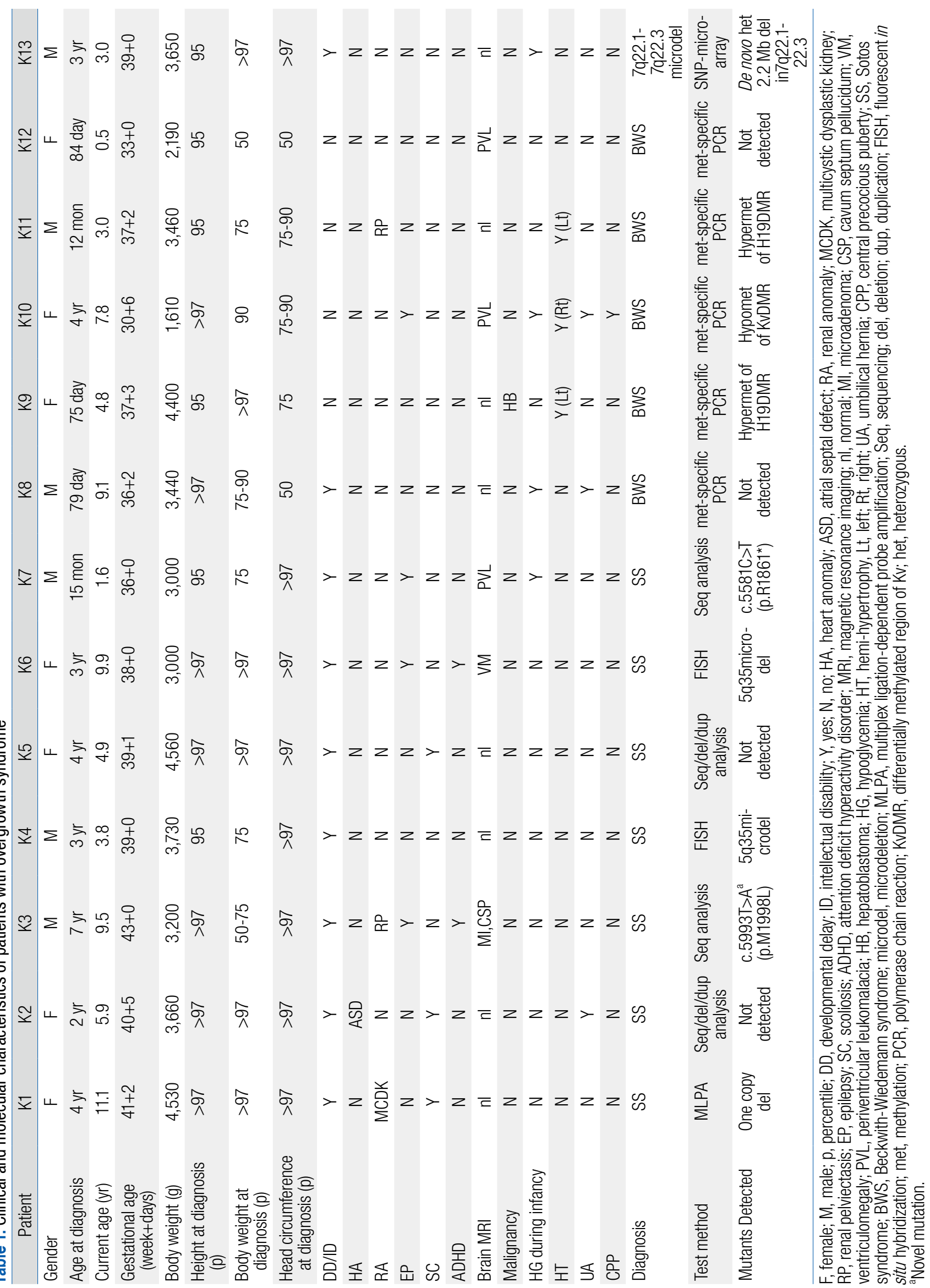




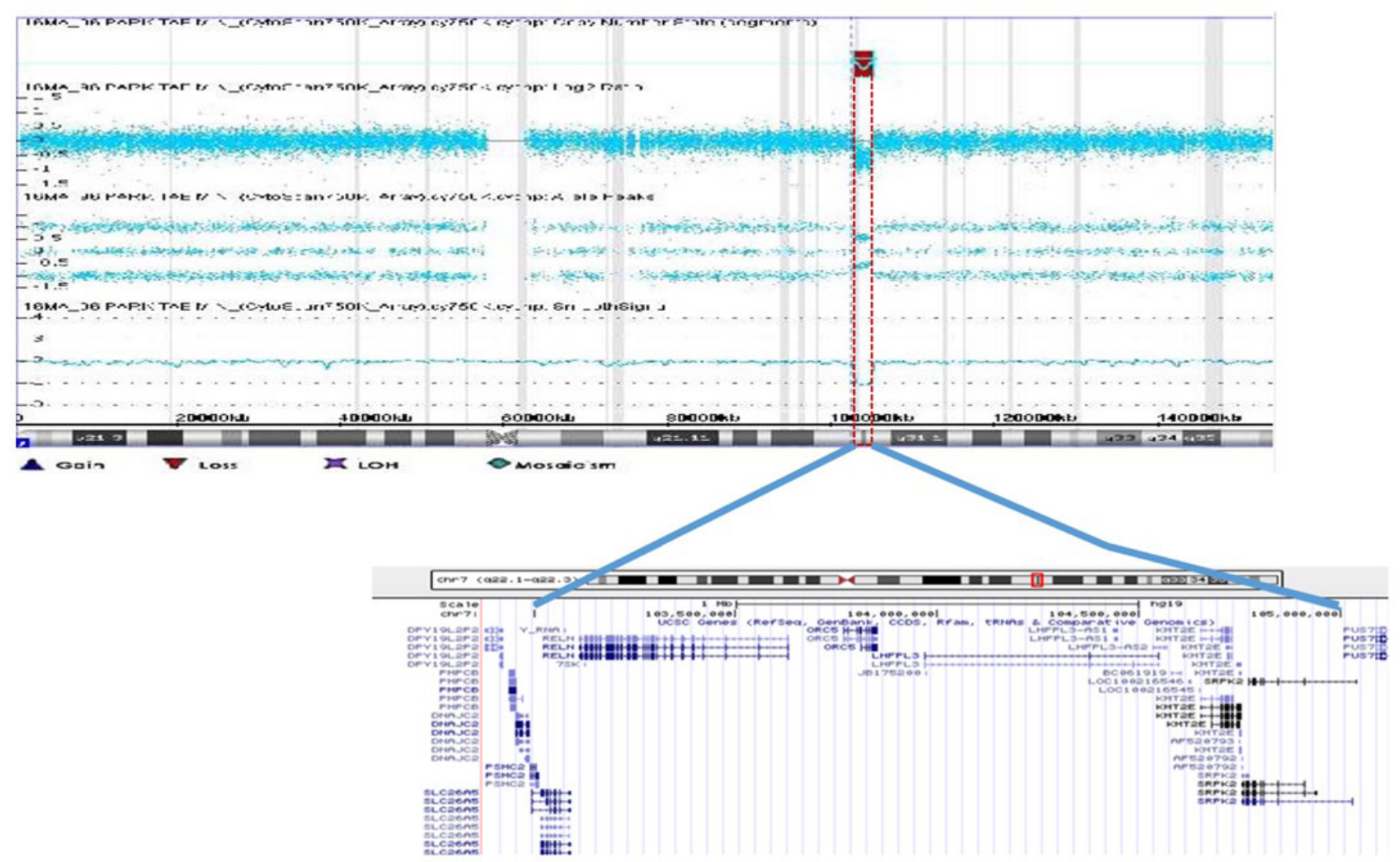

Fig. 2. Single nucleotide polymorphism-based microarray analysis plot is taken from Agilent's Analytics software (Agilent Technologies, California, CA, USA) and presented at vertical orientation. At the lower, on the chromosome 7 ideogram, the red rectangle with dotted line is indicating the position of the deletion and the affected cytoband.

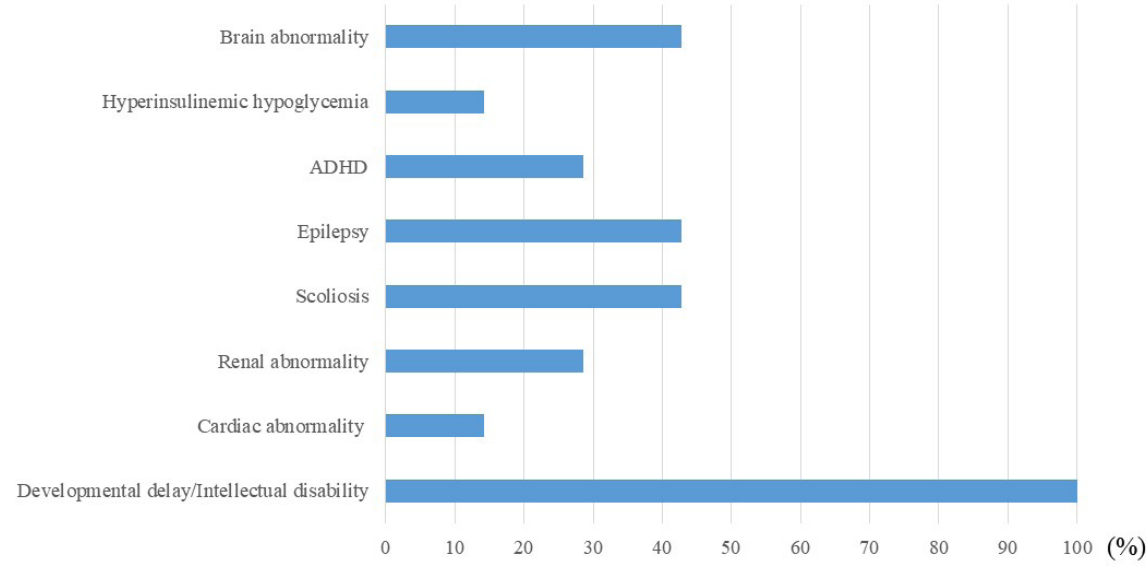

Fig. 3. Characteristics of patients with Sotos syndrome. ADHD, attention deficit hyperactivity disorder.

\section{Discussion}

This study analyzed the clinical and genetic features of 13 Korean patients with overgrowth syndrome. Among the 13 patients with overgrowth syndrome, 9 patients (69.2\%) were found to have molecular and genetic causes for overgrowth syndrome. All the patients manifested the clinical features of overgrowth syndrome, which include height $>95$ th percentile, dysmorphic craniofacial features, and other congenital anomalies. Overgrowth syndromes comprise a diverse group of clinically recognizable multiple congenital malformation syndromes [4]. Numerous multiple malformation syndromes associated with growth excess have been described. These pathologic syndromes share several characteristics, which include: overgrowth 


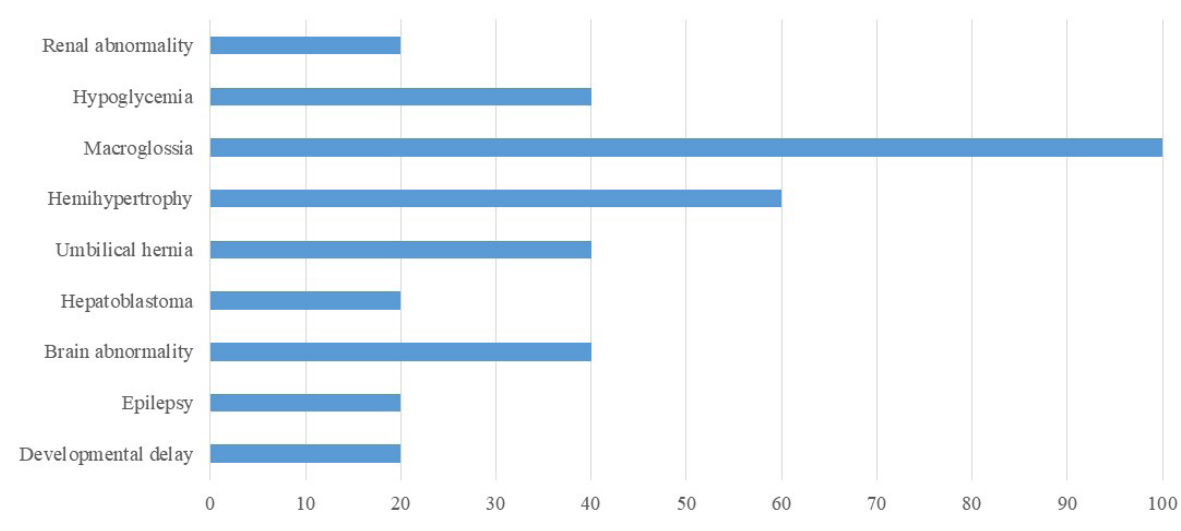

Fig. 4. Characteristics of patients with 100 (\%) Beckwith-Wiedemann syndrome.

that is present at birth and persists into postnatal life, effects on both weight and length, and an associated with multiple characteristic anomalies and often with a neoplastic predisposition [5]. For some of overgrowth syndromes including SS, ID is a key feature, although a borderline or low-normal intellect is possible. In the present study, all patients with SS showed DD or ID while one patient with BWS displayed speech delay. It is important to note that some causes of overgrowth, macrosomia, tall stature, macrocephaly, or obesity are not seen in the context of syndromes. When intellect is normal, the most common causes of overgrowth are familial, growth acceleration driven by overnutrition (including infants of diabetic mothers), and endocrine conditions such as precocious puberty, hyperthyroidism, congenital adrenal hyperplasia, growth hormone-secreting adenoma, familial glucocorticoid deficiency, and aromatase deficiency [6].

The relatively prevalent overgrowth syndromes in fetuses and neonates include SS and BWS [7]. These syndromes are not associated with overproduction of growth hormone [7]. Children with SS have distinctive facial features such as macro dolichocephaly, marked frontal bossing, and a frontoparietal receding hairline. Just over $20 \%$ of patients with SS have congenital heart defects, usually a patent ductus arteriosus or ASD [8]. In the present study, $14.2 \%$ of patients with SS had ASD, which is consistent with previous reports. Renal abnormalities and scoliosis have also been frequently reported [9]. In our study, approximately $30 \%$ of patients with SS had renal abnormalities, such as multicystic dysplastic kidney and renal pelviectasis. SS is associated with dilatation of the cerebral ventricles and other brain formation abnormalities, including an absent corpus callosum, prominent cortical sulci, trigone, occipital horns, cavum septum pellucidum, and cavum velum interposition [8]. In this study, approximately $43 \%$ of patients displayed various brain formation abnormalities that include PVL, cavum septum pel- lucidum, ventriculomegaly, and brain tumor, such as microadenoma. In addition, approximately $40 \%$ of individuals with SS reportedly experience seizures [10], which is consistent with our study (42.8\%).

Mutations in NSD 1 are responsible for SS and found in approximately $80-90 \%$ of patients [9]. SS results from constitutional intragenic loss-of-function mutations, primarily truncating mutations [3]. Of interest, $5 q 35$ microdeletions are more frequently detected in approximately 50\% of Japanese patients with SS [11], whereas $5 q 35$ microdeletions are uncommonly found less than 15\% of patients in other areas of the world [3]. In a previous report of SS in Korea, approximately 50\% of the patients had a $5 q 35$ microdeletion [12], which is slightly higher than the present finding of approximately $30 \%$. Of note, the c.5993T>A (p.M1998L) variant detected in the present study was a novel missense mutation. This region is highly conserved and this variant was not found in 1000 Genome Project, Exome Variant Server, Exome Aggregation Consortium, the dbSNP database. This variant was predicted to be deleterious through in silico analysis including SIFT (scale-invariant feature transform), polyphen-2, and Mutation taster. The patient showing this variant had intractable epilepsy and brain abnormalities such as pituitary microadenoma and cavum septum pellucidum in brain MRI. In patients with SS, brain tumors are rare. According to the large series of SS case, sacrococcygeal teratomas, presacral ganglioneuroma, neuroblastoma, and acute lymphocytic leukemia usually occurred in children with confirmed NSD 1 mutation [13]. The c.5581 C >T (p.R1861*) mutation was previously reported in 2007 [14]. The patient presented with recurrent hypoglycemic events during infancy due to hyperinsulinemic hypoglycemia, which necessitated diazoxide medication. Of interest, subject K1 had one copy deletion of NDS1 gene including FGFR4 gene. Taylor et al. [15] noted that FGFR4 is expressed in myoblasts during normal development and found that FGFR4 was significantly 
Table 2. Gene contents of the 7q22.1-22.3 deleted region

\begin{tabular}{|c|c|}
\hline Gene & Description \\
\hline PMPCB & Peptidase, mitochondrial processing, beta \\
\hline DNAJC2 & DNA/HSP40 Homolog, subfamily C, member 2 \\
\hline PSMC2 & Proteasome $26 \mathrm{~S}$ subunit, ATPase, 2 \\
\hline SLC26A5 & Solute carrier family 25 , member 5 \\
\hline$R E L N^{a}$ & Reelin \\
\hline ORC5L & $\begin{array}{l}\text { Origin recognition complex, subunit 5, Saccharomyces } \\
\text { cerevisiae, Homolog OF }\end{array}$ \\
\hline$\angle H F P L 3^{\mathrm{a}}$ & Lipoma HMGIC fusion partner-like 3 \\
\hline KMT2 & Histone-lysine N-methyltransferase 2 \\
\hline$S R P K 2^{\mathrm{a}}$ & Serine/threonine-protein kinase 2 \\
\hline
\end{tabular}

HSP, heat shock protein; ATPase, adenosine triphosphase; HMGIC, highmobility group protein isoform I-C.

a Involved in the clinical phenotype of the patient.

overexpressed in rhabdomyosarcoma tumors of high metastatic potential, and higher FGFR4 expression was associated with a lower rate of survival. In this patient, tumor surveillance might be important not only with respect to genetic counseling but also appropriate medical management.

BWS is characterized by macrosomia, asymmetric overgrowth, macroglossia, abdominal wall defects, hypoglycemia, ear creases, visceromegaly, renal malformations, facial nevus flammeus, and embryonal tumors [16]. Children with BWS have an increased risk (approximately 8.6\%) of developing embryonal tumors $[17,18]$. In the present study, one patient with BWS displayed hepatoblastoma stage II requiring left lobectomy. In BWS, the tumor risk appears to be related to the underlying defect [19]. Approximately 50\% of patients with BWS have a loss of methylation at IC2 (KvDMR1) [20]. Approximately 20\% of BWS cases have paternal uniparental disomy (pUPD) involving chromosome 11 p15 [11]. About 5-10\% of patients with BWS have a gain of methylation on the maternal IC allele, which is associated with biallelic expression of insulin-like growth factor 2 and silencing of $\mathrm{H} 19$ expression [21]. Patients with IC1 methylation alterations and pUPD11 have the highest risk of tumor $[18,20]$. In the present study, two of five patients with BWS had a gain of methylation at H19DMR, one of whom had left hepatoblastoma. Unfortunately, we could not find any abnormal methylation in two patients. Therefore, further investigation such as CDKN1C sequencing is required in the patient because sequence analysis of CDKN1C identifies 5-10\% of cases with no family history of BWS [21].

In the case of an unclassified overgrowth syndrome enrolled in the present study, overlapping clinical features of SS included high and prominent forehead, macrocephaly, and hypertelorism. However, the patient displayed severe obesity with hyperphagia, which is not consistent with the clinical manifestation of SS. Therefore, chromosomal analysis was done as the initial diagnostic work-up. The analysis result was normal. For the next step, a SNP-based microarray analysis was performed, which showed de novo heterozygous $2.2 \mathrm{Mb}$ deletion in 7q22.1-22.3. This is the first report on generalized overgrowth syndrome having the 7q22.1-7q22.3 microdeletion in Korea. Several genes have been proposed to be potentially involved in cell cycle control in 13 publications on 7q22 abnormalities. Three genes included in the presently-described microdeletion reported are involved in the clinical phenotype of the patient: LHFPL3 (Lipoma highmobility group protein isoform I-C fusion partner-like 3), SPRK2 (Serine/threonine-protein kinase 2), and RELN (Reelin) (Table 2). Computational analysis suggests that LHFPL3 protein belongs to the tetraspanin superfamily of transmembrane proteins that play an important role in the control of cell proliferation, cellular adhesion, and signaling [22]. SRPK2 belongs to the SRPK kinases, a family of cell cycle-regulated protein kinases [23]. In particular, SRPK2 directly binds to and phosphorylates acinus, upregulating its stimulatory effect on cyclin A1 [23]. Moreover, the ablation of SRPK2 or acinus arrests cell cycle at the $\mathrm{G} 1$ phase, resulting in reduced cell proliferation, whereas overexpression of acinus or SRPK2 substantially increases the G2-M phase. In our patient with the 7q22.1-7q22.3 microdeletion, the clinical findings included facial anomalies, DD, macrocephaly, hypoplasia of the corpus callosum, and cortical dysplasia. RELN is the plausible major contributor to the facial anomalies and neurological findings. During ontogenesis of the human brain, Reelin is synthesized in neurons at the telencephalic marginal zones and other pioneer neurons, as well as by granule cells of the external granular layer of the cerebellum [24]. The laminar organization of the cerebral cortex is regulated by Reelin and its receptors [25]. The central nervous system findings in our patient may be linked to hemizygous loss of RELN activity.

Taking together, clinical perception and accurate phenotyping should guide the diagnosis of more common syndromes, such as BWS and SS. However, molecular genetic confirmation and molecular classification is important, given the many types of overgrowth syndromes and because the phenotypes often overlap with patients exhibiting similar facial findings. We should consider performing chromosomal microarray for the diagnosis of unclassified overgrowth syndromes in patients where the known causes have already been excluded, because some still unclassified overgrowth syndromes may be caused by genomic imbalances. 


\section{Acknowledgements}

We thank our patient and her family members for their participation in this study. This study was supported by a 2017 research grant from Pusan National University Yangsan Hospital.

\section{References}

1. Malan V, Chevallier S, Soler G, Coubes C, Lacombe D, Pasquier L, et al. Array-based comparative genomic hybridization identifies a high frequency of copy number variations in patients with syndromic overgrowth. Eur J Hum Genet 2010;18:227-32.

2. Suri M. Approach to the diagnosis of overgrowth syndromes. Indian J Pediatr 2016;83:1175-87.

3. Tatton-Brown K, Douglas J, Coleman K, Baujat G, Cole TR, Das S, et al. Genotype-phenotype associations in Sotos syndrome: an analysis of 266 individuals with NSD1 aberrations. Am J Hum Genet 2005;77: 193-204.

4. Edmondson AC, Kalish JM. Overgrowth syndromes. J Pediatr Genet 2015;4:136-43.

5. Cohen MM Jr. Mental deficiency, alterations in performance, and CNS abnormalities in overgrowth syndromes. Am J Med Genet C Semin Med Genet 2003;117C:49-56.

6. Verge CF, Mowat D. Overgrowth. Arch Dis Child 2010;95:458-63.

7. Lebl J, Plachý L, Bláhová K, Elblová L, Fencl F, Koloušková S, et al. Overgrowth in children and in adults: novel clinical view, novel genes, novel phenotypes. Cas Lek Cesk 2017;156:233-40.

8. Tatton-Brown K, Cole TRP, Rahman N. Sotos syndrome. In: Adam MP, Ardinger HH, Pagon RA, Wallace SE, Bean $\sqcup H$, Stephens $K_{1}$ et al, eds. GeneReviews $^{\circledR}$ [Internet]. Seattle (WA): University of Washington, 2004 Dec 17 [Updated 2015 Nov 19; cited 2018 May 25]. [https:// www.ncbi.nlm.nih.gov/books/NBK1479/]

9. Tatton-Brown K, Rahman N. The NSD1 and EZH2 overgrowth genes, similarities and differences. Am J Med Genet C Semin Med Genet 2013;163C:86-91.

10. Nicita F, Ruggieri M, Polizzi A, Mauceri L, Salpietro V, Briuglia S, et al. Seizures and epilepsy in Sotos syndrome: analysis of 19 Caucasian patients with long-term follow-up. Epilepsia 2012;53:e102-5.

11. Kurotaki N, Imaizumi K, Harada N, Masuno M, Kondoh T, Nagai T, et al. Haploinsufficiency of NSD1 causes Sotos syndrome. Nat Genet 2002;30:365-6.

12. Sohn $Y B$, Lee $C G$, Ko JM, Yang JA, Yun JN, Jung EJ, et al. Clinical and genetic spectrum of 18 unrelated Korean patients with Sotos syndrome: frequent $5 q 35$ microdeletion and identification of four novel
NSD1 mutations. J Hum Genet 2013;58:73-7.

13. Tatton-Brown K, Rahman N. Clinical features of NSD1-positive Sotos syndrome. Clin Dysmorphol 2004;13:199-204.

14. Saugier-Veber $P_{1}$ Bonnet $C$, Afenjar $A$, Drouin-Garraud V, Coubes $C$, Fehrenbach $S$, et al. Heterogeneity of NSD1 alterations in 116 patients with Sotos syndrome. Hum Mutat 2007;28:1098-107.

15. Taylor JG 6th, Cheuk AT, Tsang PS, Chung JY, Song YK, Desai $K$, et al. Identification of FGFR4-activating mutations in human rhabdomyosarcomas that promote metastasis in xenotransplanted models. J Clin Invest 2009;119:3395-407.

16. Choufani S, Shuman C, Weksberg R. Molecular findings in BeckwithWiedemann syndrome. Am J Med Genet C Semin Med Genet 2013; 163C:131-40.

17. Tan TY, Amor DJ. Tumour surveillance in Beckwith-Wiedemann syndrome and hemihyperplasia: a critical review of the evidence and suggested guidelines for local practice. J Paediatr Child Health 2006; 42:486-90.

18. Brioude F, Lacoste A, Netchine I, Vazquez MP, Auber F, Audry G, et al. Beckwith-Wiedemann syndrome: growth pattern and tumor risk according to molecular mechanism, and guidelines for tumor surveillance. Horm Res Paediatr 2013;80:457-65.

19. Mussa A, Russo S, De Crescenzo A, Freschi A, Calzari L, Maitz S, et al. (Epi)genotype-phenotype correlations in Beckwith-Wiedemann syndrome. Eur J Hum Genet 2016;24:183-90.

20. Weksberg R, Shuman C, Beckwith JB. Beckwith-Wiedemann syndrome. Eur J Hum Genet 2010;18:8-14.

21. Ibrahim A, Kirby G, Hardy C, Dias RP, Tee L, Lim D, et al. Methylation analysis and diagnostics of Beckwith-Wiedemann syndrome in 1,000 subjects. Clin Epigenetics 2014;6:11.

22. Ptacek $T$, Song $C$, Walker CL, Sell SM. Physical mapping of distinct $7 q 22$ deletions in uterine leiomyoma and analysis of a recently annotated 7q22 candidate gene. Cancer Genet Cytogenet 2007;174:11620.

23. Jang SW, Yang SJ, Ehlén A, Dong $S$, Khoury $H$, Chen J, et al. Serine/ arginine protein-specific kinase 2 promotes leukemia cell proliferation by phosphorylating acinus and regulating cyclin A1. Cancer Res 2008;68:4559-70.

24. Hiesberger $T$, Trommsdorff $M$, Howell BW, Goffinet $A$, Mumby MC, Cooper JA, et al. Direct binding of Reelin to VLDL receptor and ApoE receptor 2 induces tyrosine phosphorylation of disabled-1 and modulates tau phosphorylation. Neuron 1999;24:481-9.

25. Dulabon L, Olson EC, Taglienti MG, Eisenhuth S, McGrath B, Walsh $\mathrm{CA}$, et al. Reelin binds alpha3beta 1 integrin and inhibits neuronal migration. Neuron 2000;27:33-44. 